\title{
Compressed-Sampling-Based Behavioural Modelling Technique for Wideband RF Transmitter Leakage Cancellation System
}

\author{
Han Su, Ziming Wang, Ronan Farrell \\ Department of Electronic Engineering, \\ National University of Ireland, Maynooth \\ Email: hsu@eeng.nuim.ie
}

\begin{abstract}
A duplexer is necessary, but unfavourable for a frequency-division duplexing (FDD) base station, due to its bulky size, high cost and design challenges. In order to relax the performance requirement of such device, the transmitter (TX) leakage needs to be suppressed. The state-of-the-art solutions failed to provide a wideband solution for cancelling the TX leakage at RF frequency, due to the lack of delay optimization. Aiming to provide high delay estimation accuracy, this paper presents a modelling technique which is based on the compressed sampling matching pursuit (CoSaMP) algorithm for compressed sampling (CS). As a result, by using the proposed modelling technique, cancellation systems, particularly the ones that are based on the analog finite impulse response (FIR) filter structure, can be implemented to achieve wideband suppressing at RF frequencies.
\end{abstract}

Index Terms-Self-Interference, CoSaMP, Compressed sampling (CS), Behavioural modelling, Leakage cancellation, Analog FIR filter

\section{INTRODUCTION}

In a frequency-division duplexing (FDD) base station, signals of the transmitter (TX) and receiver (RX) are separated by a device known as a duplexer. The duplexer needs to provide great isolation between the TX and RX ports which makes such device bulky, expensive and difficult to design. It is desirable for the industries to relax the high isolation performance requirement of the duplexer. In order to do so, the problem that is due to the reduced isolation between TX and RX needs to be solved.

Due to the lower isolation, the TX signal along with the noise floor and distortions of the high power amplifier (PA) appears in the RX path. Such signal is known as TX leakage and can be considered as in-band and out-of-band portions with respect to the RX band. Theoretically, the out-of-band portions can be eliminated utilising a filter. The in-band portion is more problematic as it can't be filtered out using conventional filtering techniques. As a result, RX performance is reduced. This implies the need of non-conventional techniques in order to cancel the TX leakage.

Many researchers are keen to suppress the in-band TX leakage using active cancellation techniques. Techniques that are proposed in [2] [3] [4] are based on the analog finite impulse response (FIR) filter structure. These cancellation techniques consist of a number of auxiliary cancellation paths (also known as taps). In each of the paths, the delayed replica of the reference signal is attenuated and phase-shifted. These reconditioned replicas of the reference signal are combined to form the cancellation signal. Since the cancellation signal in theory has the same amplitude and $180^{\circ}$ out of phase from the leakage signal, by summing the two, the leakage is suppressed. Most of the techniques such as the ones in [2] [3] [4] have abilities to optimize the phase and amplitudes, but not the delays. Since FDD duplexers usually have complex characteristics, i.e., the magnitude and group delay response change rapidly and the phase response is highly non-linear, these techniques are inherently for narrowband cancellation, or else significantly more hardware resources are required for cancellation over wide bandwidth. Therefore, a modelling technique that can provide optimization of delays is essential for successfully cancelling the in-band TX leakage over wide bandwidth.

The work in [6] which is also based on the analog FIR filter structure has presented a methodology for wideband cancellation. It involves a modelling technique that can provide delay optimization. However, this modelling technique is less robust and can potentially be trapped in an endless loop due to the lack of phase control ability.

In this paper, we propose a modelling technique that is designed for the wideband RF cancellation system that is based on the analog FIR filter structure. It aims to provide the optimization ability of the delays, amplitude and phase, in order to achieve better cancellation performance. At the same time the least hardware resources are required. The proposed technique is based on the classic compressed sampling matching pursuit (CoSaMP) algorithm. This paper firstly introduces the CoSaMP algorithm. The problems of using the classic CoSaMP are discussed and the solution is presented. For evaluation purposes, an FDD transceiver test bed with an off-the-shelf ceramic duplexer has been constructed. The modelling performance is produced using the signals that are captured by the transceiver.

\section{Introduction to COSAMP Algorithm}

CoSaMP is a compressed sampling (CS) technique that was originally developed in order to more efficiently solve the under-determined systems, providing that those systems have 
sparse nature. Let $\mathbf{y}$ be the vector of the measured output, the size of which is $M-b y-1$. Mathematically, it can be represented as

$$
\mathbf{y}=X \mathbf{w}
$$

where the size of the model matrix $X$ is $M-b y-N$, providing that $M \ll N$, and the coefficients vector $\mathbf{w}(N-b y-1)$ is sparse. The input matrix $X$ in this work is the toeplitz matrix of the input reference signal $x(n)$, i.e.:

$$
X=[x(n), x(n-1), \ldots, x(n-M)]
$$

where $x(n)$ is a $N-b y-1$ vector. Traditional CS algorithms tend to approximate the sparse systems by obtaining the coefficients vector $\hat{\mathbf{w}}$, which satisfies the condition of

$$
\min \|\hat{\mathbf{w}}\|_{l_{1}} \text { subject to }\|X \hat{\mathbf{w}}-\mathbf{y}\|_{l_{2}}<\varepsilon
$$

where $\varepsilon$ is the desired tolerance of the error.

Rather than reducing the number of measurement samples, CS can be utilised for reducing the number of coefficients of a sparse system. This requires that the sizes of the input matrix $X$, the output signal of the system $\mathbf{y}$ and the coefficients vector w are $N-b y-M, N-b y-1$ and $M-b y-1$, respectively, providing that $\mathbf{w}$ is $k$-sparse. $k$-sparse means that the vector $\mathbf{w}$ consists of $k$ non-zero components. Typical applications of CS in terms of reducing the number of coefficients are behavioural modelling and digital pre-distortion of high power amplifiers in wireless communications [1]. Comparing to the traditional $\mathrm{CS}$ algorithms, CoSaMP is more efficient and less complex.

CoSaMP was developed in [5] and is based on the orthogonal matching pursuit (OMP) algorithm. It approximates the coefficients vector $\mathbf{w}$ iteratively by obtaining the sparse representation $\tilde{\mathbf{w}}$ and calculating the residual error. CoSaMP algorithm has the advantage of low-complexity and provides faster speed and stronger guarantees than the original OMP [5] . CoSaMP algorithm requires some information including: the input model matrix, the desired signal vector, the desired sparsity of the approximation and the stopping condition. The algorithm estimates the location of the most significant tap weights iteratively. The algorithm is ended while stopping criterion is reached. Upon the completion of the standard CoSaMP algorithm, the coefficients and the locations of the most significant taps are determined. During the initialization, input model matrix of the system is created. The desired sparsity $k$ and the tolerance $\varepsilon$ are also provided. The residue signal from the previous iteration is set to be the output vector y.

The details of the operation procedures after the initialization are summarised as follows and these procedures repeat until stopping criteria is reached:

1) Identification : Approximating the residue signal using input matrix and determining the locations of the largest $2 k$ coefficients.

2) Merging: Merging the locations of the determined largest $2 k$ coefficients with the ones that are obtained by the previous iteration.
3) Estimation: Re-calculating the coefficients at the merged locations.

4) Pruning: Choosing the locations of the largest $k$ coefficients for the next iteration.

5) Sample Updating : Approximating the desired signal $\mathbf{y}$ utilizing the input matrix and determined locations of the coefficients. The error between the desired and the approximated signals is updated as the residue for the next iteration.

In this work, the approximating procedures utilize the least squares (LS) algorithm due to the low implementation complexity and high modelling performance.

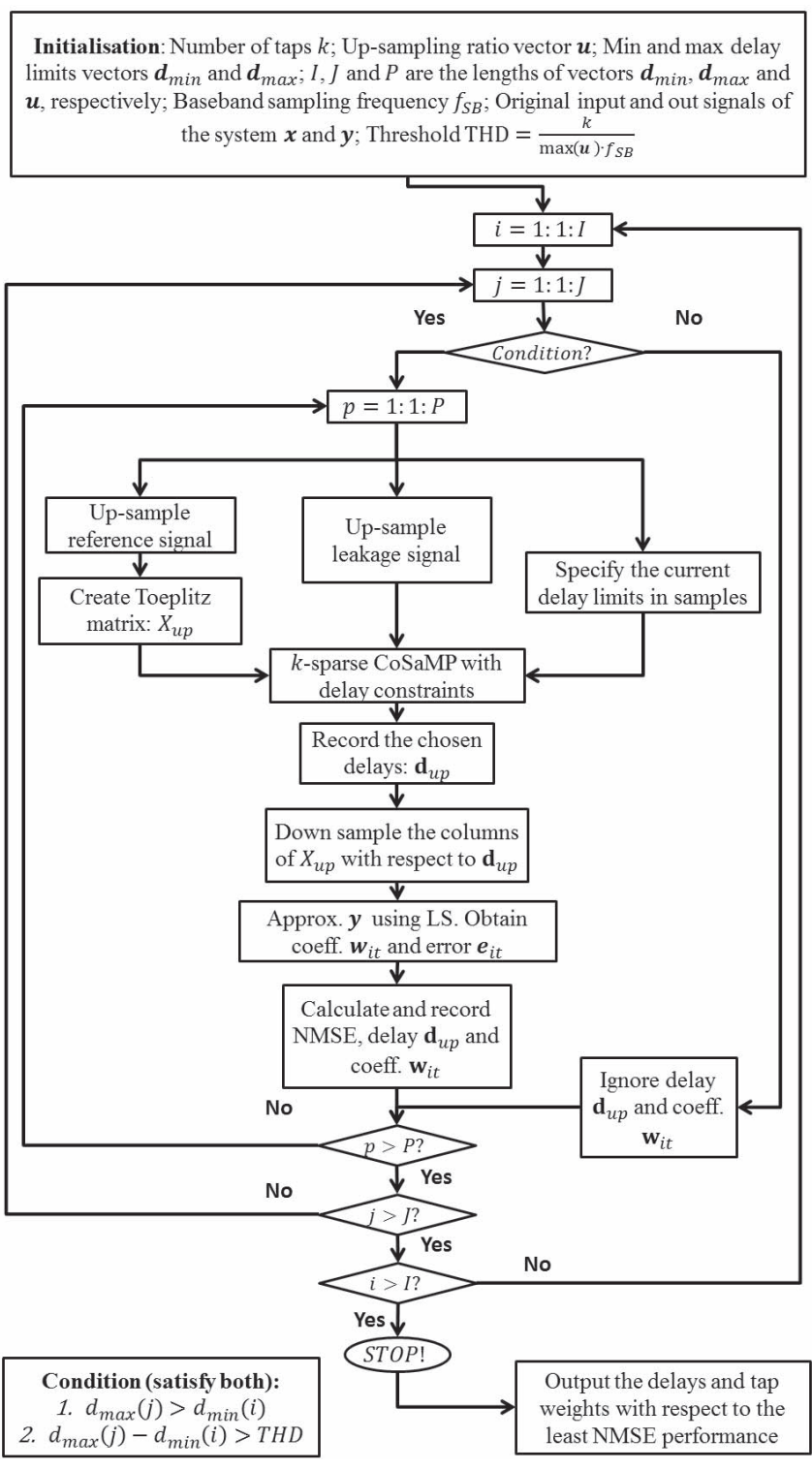

Fig. 1. Flowchart of the proposed modelling technique

\section{The Adaptation of CoSAMP FOR TX LEAKAgE CANCELlation}

One key output using CoSaMP is a set of locations at which the coefficients are the most significant. In the TX leakage 
cancellation using the analog FIR structure, these locations represent the desired delays of the cancellation paths (taps) at $\mathrm{RF}$ frequency. The resolution $\tau_{0}$ of these delays is the sampling time $\tau_{s}$ of the baseband signals, i.e.:

$$
\begin{aligned}
\tau_{0} & =\tau_{s} \\
& =\frac{1}{f_{s}}
\end{aligned}
$$

where $f_{s}$ is the sampling frequency at which the delays are estimated. Since the sampling frequency of the reference and leakage signals at the baseband is $f_{S B}$, the delay resolution thus is $\tau_{0}=\frac{1}{f_{S B}}$. Because we are only interested in the cancellation bandwidth that is a fraction of the carrier frequency, we can safely assume that the RF signals are sampled at an extremely high frequency and the impulse response of the isolation path of the duplexer is formed at the same sampling rate. Then we notice that the delay resolution at the baseband using CoSaMP is insufficient for implementing the cancellation system at RF frequency. Using CoSaMP under such low delay resolution could result the increase of the number of cancellation paths or the decrease of the cancellation performance.

By up-sampling the baseband signals with a ratio, a higher sampling frequency can be achieved and thus greater delay resolution can be obtained. The exact value of the up-sampling ratio which results the least modelling error is unknown. We propose that rather than giving an exact value, the up-sampling ratio is swept within a given range. The one that gives the least modelling error is chosen for the implementation of the cancellation system.

Since the delays that are obtained by modelling, would be used in analog domain at RF frequency, limiting these delays within a range is essential so that the estimated delays are implementable. Additionally, through excessive experiments, we found out that sweeping the minimum and maximum values of the delay limit ( $\mathbf{d}_{\min }$ and $\mathbf{d}_{\max }$, respectively) in addition to sweeping the up-sampling ratio can potentially further reduce the modelling error. As a result, the proposed modelling technique utilizes the classic CoSaMP algorithm and sweeps through the vectors of up-sampling ratios and delay limits. The detailed procedures of the proposed modelling technique are shown in Fig. 1 and Fig. 2.

Let the vector of the up-sampling ratios be $\mathbf{u}$. Before sweeping through $\mathbf{u}$ and estimating the system with the CoSaMP kernel, the validity of the current values of the delay limit vectors, i.e., $\mathbf{d}_{\min }(i)$ and $\mathbf{d}_{\max }(j)$ must be checked first, where $i=1,2, \ldots, I$ and $j=1,2, \ldots, J$ are sweeping indexes for the vector of minimum and maximum delays, respectively. The values $I$ and $J$ are the number of elements in $\mathbf{d}_{\min }$ and $\mathbf{d}_{\max }$, respectively. This is to ensure that $\mathbf{d}_{\min }(i)<\mathbf{d}_{\max }(j)$ and $\mathbf{d}_{\max }(j)-\mathbf{d}_{\min }(i)>T H D$ where the delay threshold value $T H D=\frac{k}{\max (\mathbf{u}) f_{S B}}$ is the minimum range in which the locations of all $k$ taps are valid. For any $\mathbf{u}(p)$ where $p=1,2, \ldots, P$ is the sweeping index and $P$ is the length of the vector $\mathbf{u}$, the original input reference vector $\mathbf{x}=x(n)$ and leakage signals $\mathbf{y}$ are up-sampled accordingly. Thus the

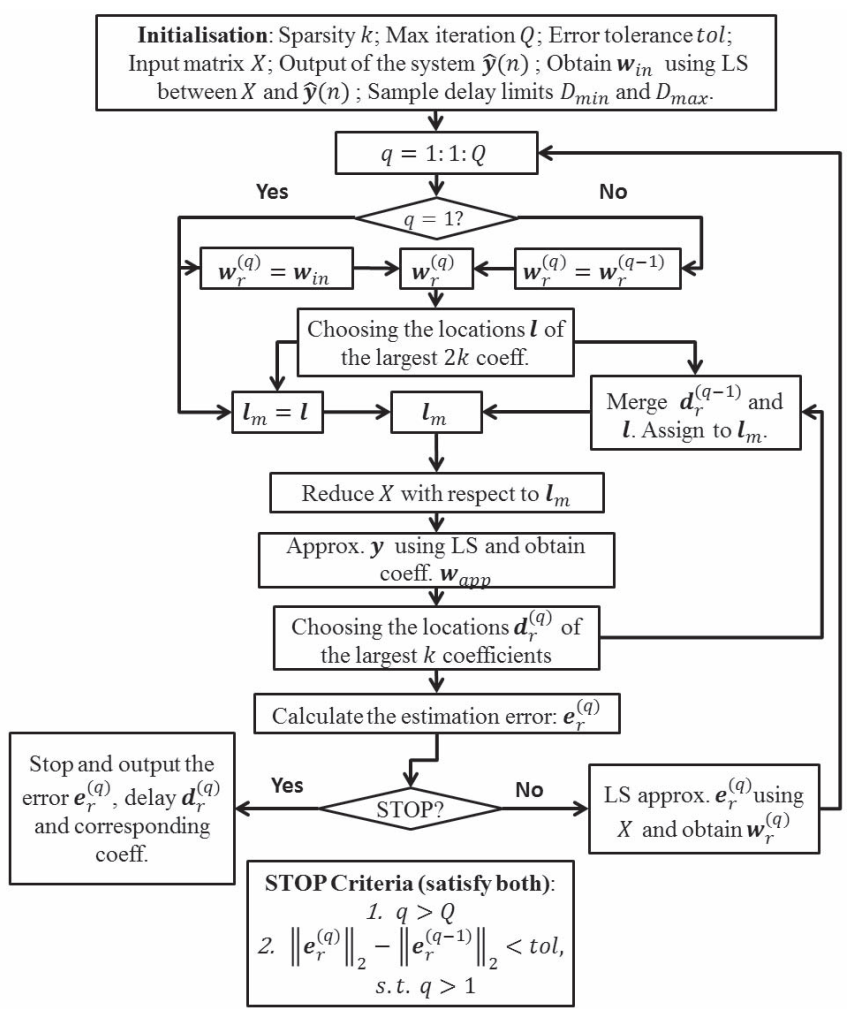

Fig. 2. Flowchart of the classic CoSaMP with delay constraints

input matrix $X_{u p}$ can be created with the up-sampled reference vector $\mathbf{x}_{u p}$. With $X_{u p}$ and the up-sampled leakage signal $\mathbf{y}_{u p}$, the system can be modelled utilizing the CoSaMP algorithm.

The kernel of the classic CoSaMP needs to be modified so that the delay limits can take effect. The flowchart of the modified CoSaMP is shown in Fig. 2. The delay constraints in samples, i.e., $D_{\min }$ and $D_{\max }$ are included as two additional input parameters to the classic CoSaMP technique. During the identification and pruning procedures of the classic CoSaMP algorithm, the locations of the most significant coefficients must be chosen within the range that is bounded by $D_{\min }$ and $D_{\max }$.

The locations of the most significant coefficients are determined by the modified CoSaMP kernel. Let these locations be $\mathbf{l}_{m}$. The vectors, $X_{u p}\left(:, \mathbf{l}_{m}\right)$ are down-sampled to $f_{S B}$ and formed into a new matrix, i.e., $X_{C S}=\operatorname{Dn} \operatorname{Samp}\left(X_{u p}\left(:, \mathrm{l}_{m}\right)\right)$. The leakage signal $\mathbf{y}$ is approximated with $X_{C S}$ using LS algorithm. Along with the delays $\mathbf{l}_{m}$, the resulting tap weights and normalised mean square error (NMSE) of the remaining error signal are recorded, separately. The above procedures are repeated until all elements of $\mathbf{u}, \mathbf{d}_{\min }$ and $\mathbf{d}_{\max }$ vectors are swept. The up-sampling ratio, minimum and maximum delay limits that produce the least NMSE are chosen and the corresponding tap weights and delays are the ones for implementing the cancellation system.

\section{Validation of the Proposed Technique}

For the purpose of validating the proposed modelling technique, a transceiver test bench has been set up which is shown 
in Fig. 3. The transmitter consists of a vector signal generator (SMU by Rohde \& Schwarz), a high power amplifier (1184BBM3O6QCK from Empower RF systems) and a bandpass filter. The TX signal is created with a $35 \mathrm{MHz}$ white Gaussian noise signal at centre frequency of $897.5 \mathrm{MHz}$. A ceramic duplexer (SCD900AE4 from Samnova) is used as the duplexing device of this transceiver. The TX signal is coupled off via two directional couplers. A high speed oscilloscope (Agilent 54853A) is used to capture and digitize the input reference and the leakage signals. These two signals are digitally frequency down-converted to baseband using MATLAB. In practice, the cancellation block is controlled by the PC. It is located between the $30 \mathrm{~dB}$ coupler at the auxiliary path and the two way power combiner at the main receiver. The implementation of the cancellation block is outside the scope of this paper.

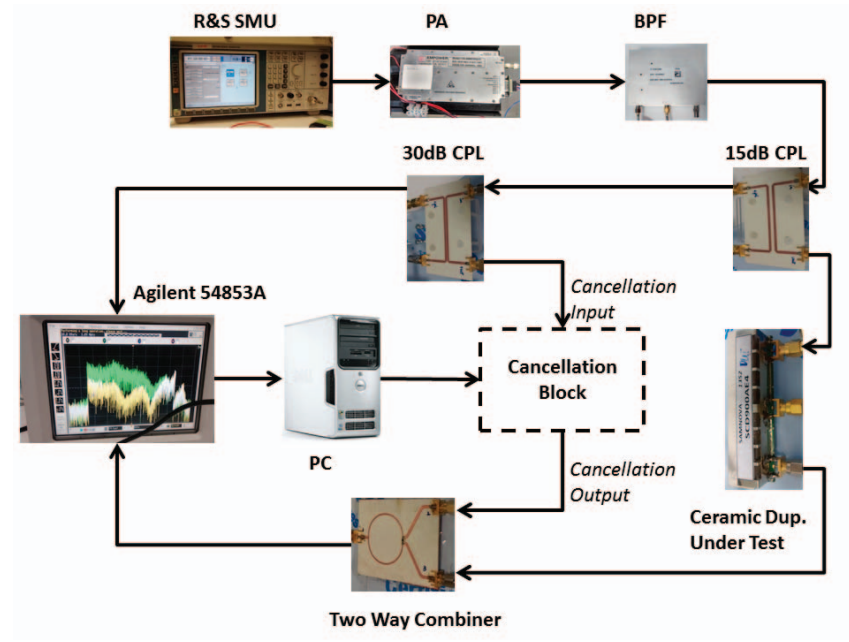

Fig. 3. Measurement setup

Due to the limited reflection performance and high distortion characteristics of the high power amplifier, the reference signal that is captured by the auxiliary receiver and the input signal of the duplexer are no long white. In order to achieve the best performance, the baseband reference signal is passed through a whitening filter. The coefficients of the whitening filter are also applied to the leakage signal. Once the modelling of the duplexer is completed and the delays and coefficients are obtained, these parameters are applied to the actual reference signal in order to obtain the approximation signal. The error between the approximated signal and the original leakage is displayed in Fig. 4. The result also includes the original leakage and the error signals using the classic CoSaMP and LS algorithms. All three techniques are evaluated using four taps. The remaining errors that are obtained by the classic CoSaMP and LS are very similar. The proposed technique can achieve approximately $-21 \mathrm{~dB}$ of NMSE for $35 \mathrm{MHz}$ of bandwidth, whereas more than $-10 \mathrm{~dB}$ has been achieved using the classic CoSaMP. This result implies that the estimated delays of the proposed modelling technique can more accurately estimate the delays. This is critical for implementing the wideband RF cancellation system using analog FIR filter structure.

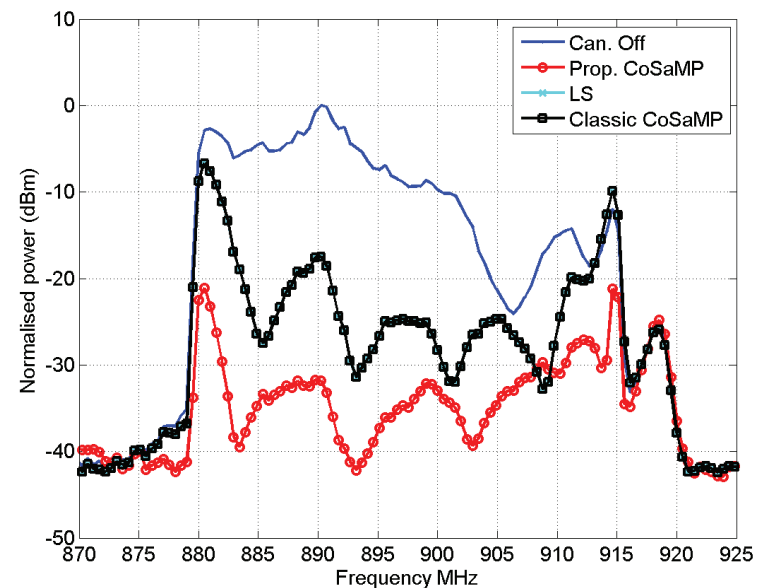

Fig. 4. Modelling error comparison

\section{CONCLUSION}

For the purpose of implementing the wideband TX leakage cancellation system so that the requirement of the high isolation performance FDD duplexers can be relaxed, a modelling technique is proposed in this paper. The modelling technique takes advantages of CoSaMP algorithm and is modified to provide higher delay estimation accuracy. The proposed technique is evaluated using the signals that are captured with a transceiver test bed. The result shows that the proposed modelling technique outperforms the classic CoSaMP algorithm and is suitable for implementing the desired TX leakage cancellation system.

\section{ACKNOWLEDGMENT}

This material is based upon works supported by the Science Foundation Ireland under Grant No. 10/CE/I1853 as part of the Centre for Telecommunications Research (CTVR). The authors gratefully acknowledge this support.

\section{REFERENCES}

[1] A. Abdelhafiz, A. Kwan, O. Hammi, and F.M. Ghannouchi. Digital predistortion of lte-a power amplifiers using compressed-sampling-based unstructured pruning of volterra series. Microwave Theory and Techniques, IEEE Transactions on, 62(11):2583-2593, Nov 2014.

[2] Yingbo Hua, Yiming Ma, Armen Gholian, Yifan Li, Ali Cagatay Cirik, and Ping Liang. Radio self-interference cancellation by transmit beamforming, all-analog cancellation and blind digital tuning. Signal Processing, 108:322 - 340, 2015.

[3] Hyoungsoo Kim, Sanghyun Woo, Sungyong Jung, and Kil-Hoon Lee. A cmos transmitter leakage canceller for wcdma applications. Microwave Theory and Techniques, IEEE Transactions on, 61(9):3373-3380, 2013.

[4] S. Maddio, A. Cidronali, A. Palonghi, and G. Manes. A reconfigurable leakage canceler at $5.8 \mathrm{ghz}$ for dsrc applications. In Microwave Symposium Digest (IMS), 2013 IEEE MTT-S International, pages 1-3, June 2013.

[5] D. Needell and J.A. Tropp. Cosamp: Iterative signal recovery from incomplete and inaccurate samples. Applied and Computational Harmonic Analysis, 26(3):301 - 321, 2009.

[6] Han Su, G. Szczepkowski, and R. Farrell. Wideband tx leakage cancellation using adaptive delay filter at $\mathrm{rf}$ frequencies. In Irish Signals Systems Conference 2014 and 2014 China-Ireland International Conference on Information and Communications Technologies (ISSC 2014/CIICT 2014). 25th IET, pages 396-401, June 2014. 